\title{
Polyphenols from Korean prostrate spurge Euphorbia supina induce apoptosis through the Fas-associated extrinsic pathway and activation of ERK in human leukemic U937 cells
}

\author{
MIN-HO HAN ${ }^{1}$, WON SUP LEE ${ }^{2}$, ARULKUMAR NAGAPPAN ${ }^{2}$, HYE JUNG KIM $^{3}$, CHEOL PARK $^{4}$, \\ GI-YOUNG KIM ${ }^{5}$, SANG HOON HONG ${ }^{6}$, NAM DEUK KIM ${ }^{7}$, GONSUP KIM ${ }^{8}$, CHUNG HO RYU $^{9}$, \\ SUNG CHUL SHIN ${ }^{10}$ and YUNG HYUN CHOI ${ }^{11}$
}

\begin{abstract}
${ }^{1}$ Natural Products Research Team, National Marine Biodiversity Institute of Korea, Seocheon 325-902;
Departments of ${ }^{2}$ Internal Medicine, and ${ }^{3}$ Pharmacology, Institute of Health Sciences, Gyeongsang National University School of Medicine, Jinju 660-702; ${ }^{4}$ Department of Molecular Biology, College of Natural Sciences and Human Ecology, Dongeui University, Busan 614-714; ${ }^{5}$ Department of Marine Life Sciences, Jeju National University, Jeju 690-756;

${ }^{6}$ Department of Internal Medicine, Dongeui University College of Korean Medicine, Busan 614-052;

${ }^{7}$ Department of Pharmacy, College of Pharmacy, Pusan National University, Busan 609-735;

${ }^{8}$ School of Veterinary Medicine, ${ }^{9}$ Division of Applied Life Science (BK 21 Program) and ${ }^{10}$ Department of Chemistry, Research Institute of Life Science, Gyeongsang National University, Jinju 660-701; ${ }^{11}$ Department of Biochemistry, Dongeui University College of Korean Medicine, Busan 614-052, Republic of Korea
\end{abstract}

Received February 1, 2016; Accepted March 10, 2016

DOI: $10.3892 /$ or.2016.4778

\begin{abstract}
The Korean prostrate spurge Euphorbia supina (Euphorbiaceae family) has been used as a folk medicine in Korea against a variety of ailments such as bronchitis, hemorrhage, jaundice and multiple gastrointestinal diseases. Polyphenols from Korean E. supina (PES) which include quercetin and kaempferol derivatives have anticancer properties. Hence, we investigated the anticancer effects of PES on U937 human leukemic cells. Firstly, PES significantly inhibited the proliferation of U937 cells in a dose-dependent manner. PES induced accumulation of the sub-G1 DNA content (apoptotic cell population), apoptotic bodies and chromatin condensation and DNA fragmentation in the U937 cells. PES also induced activation of caspase- $3,-8$ and -9 , subsequent cleavage of PARP, and significantly suppressed XIAP, cIAP-1 and cIAP-2 in a dose-dependent manner. Furthermore, PES activated Bid, and
\end{abstract}

Correspondence to: Professor Won Sup Lee, Department of Internal Medicine, Institute of Health Sciences and Gyeongnam Regional Cancer Center, Gyeongsang National University School of Medicine, and Gyeongsang National University Hospital, 90 Chilam-dong, Jinju 660-702, Republic of Korea

E-mail: lwshmo@hanmail.net; lwshmo@gnu.ac.kr

Professor Yung Hyun Choi, Department of Biochemistry, Dongeui University College of Oriental Medicine, 42 San, Yangjung-dong, Busan 614-052, Republic of Korea

E-mail: choiyh@deu.ac.kr

Key words: Euphorbia supina, polyphenols, apoptosis, Fas receptor, extracellular signal-regulated kinase pathway, U937 human leukemic cells induced the loss of mitochondrial membrane potential (MMP, $\Delta \Psi \mathrm{m}$ ) along with upregulation of pro-apoptotic proteins (Bax and $\mathrm{Bad}$ ), and downregulation of anti-apoptotic proteins (Bcl-2 and $\mathrm{Bcl}-\mathrm{xL}$ ) and cytochrome $c$ release. The Fas receptor was upregulated by PES in a dose-dependent manner, suggesting that the extrinsic pathway was also involved in the PES-induced apoptosis. Moreover, the PES-induced apoptosis was at least in part associated with extracellular signal-regulated kinase (ERK) activation in the U937 human leukemic cells. This study provides evidence that PES may be useful in the treatment of leukemia.

\section{Introduction}

Considerable interest has been drawn to the possibility of preventing or controlling cancer using phytochemicals since phytochemicals can safely modulate cancer cell biology and induce cancer cell death. In addition, natural herbs have become more popular in preventing or controlling cancer as alternative therapy due to the following reasons. Firstly, the high intake of fruit and vegetables containing abundant polyphenols is associated with a low incidence of cancer $(1,2)$. Secondly, phytochemicals from natural products or herbs show anticancer effects without noticeable toxicities (3). Thirdly, the number of elderly cancer patients who cannot tolerate increasingly intense conventional chemotherapy is increasing $(4,5)$. The Korean prostrate spurge Euphorbia supina (E. supina) belongs to the Euphorbiaceae family. It has been used in folk medicine in Korea against a variety of ailments such as bronchitis, hemorrhage, jaundice and multiple gastrointestinal diseases (6). It contains a number of biologically active substances $(7,8)$. Among them, polyphenols have attracted our interest due to their beneficial effects on human health. Literature has shown that polyphenols 
reduce the risk of chronic diseases and possess antioxidant and anti-aging properties (9). It was reported that Korean E. supine is abundant in polyphenols. Song et al (6) isolated and identified nine polyphenols from Korean E. supine (PES) which included quercetin and kaempferol derivatives which have anticancer properties.

Evidence suggests that the major mechanism for the anticancer effects of polyphenols is apoptosis, a type I programmed cell death showing a distinctive phenotype: cytoplasmic blebbing, nuclear condensation and fragmentation, and DNA fragmentation $(10,11)$. Polyphenol- or phytochemical-induced apoptosis are mostly caspase-dependent. This caspase-induced apoptosis is carried out through the intrinsic pathway and/or extrinsic pathway (12-15).

The intrinsic apoptotic pathway involves non-receptormediated caspase activation through intracellular signals which increase permeability of the mitochondria and facilitate release of cytochrome $c$ into the cytoplasm. The cytochrome $c$ then initiates activation of the caspase cascade through caspase-9 (16). The extrinsic apoptotic pathway involves death receptor-mediated caspase activation. The death receptors such as tumor necrosis factor receptor 1 (TNFR1) and FAS receptor (Fas)/CD95 are on the plasma membrane $(17,18)$. Through increase of ligands or their receptors, the deathinducing signaling complex (DISC) is formed as ligands bind to these receptors, and then the caspase cascade is activated through caspase-8 (19).

The Bcl-2 family consists of pro-apoptotic and anti-apoptotic proteins involved in permeability of the mitochondrial membrane $(16,20)$. In addition, the signaling pathways of mitogen-activated protein kinases (MAPKs) and protein kinase $\mathrm{B}$ (PKB or $\mathrm{Akt}$ ) are involved in directing cellular responses to a diverse array of stimuli, and regulate cell survival and apoptosis $(21,22)$. However, the underlying mechanisms of apoptosis induced by polyphenols from PES against human leukemic cells have not been reported yet.

Hence, the aim of the present study was to investigate the mechanisms involved in the anticancer effects of PES on U937 human leukemic cells. Our data suggest that PES induced apoptosis via both the intrinsic and extrinsic apoptotic signaling pathways; PES upregulated Fas, activated Bid, and suppressed $\mathrm{CFLIP}_{\mathrm{L}}$ and Bcl-xL modulation of the Bcl-2 family and the inhibitor of apoptosis protein (IAP) family proteins, finally inducing apoptosis through activation of caspases. In addition, the PES-induced apoptosis was at least in part associated with extracellular signal-regulated kinase (ERK) activation. This is the first study demonstrating the anticancer effects of PES against U937 human leukemic cells.

\section{Materials and methods}

Preparation of PES. PES were extracted and purified by Professor S.C. Shin (6). Briefly, the lyophilized E. supina tissue $(10 \mathrm{~g})$ was extracted in ethyl acetate $(300 \mathrm{ml})$ at $80^{\circ} \mathrm{C}$ for $20 \mathrm{~h}$, and eluted using a mixture of methanol:dichloromethane (1:5, $25 \mathrm{ml})$. The isolated polyphenol mixtures were identified by HPLC-MS/MS. The nine PES were identified: gallic acid, protocatechuic acid, nodakenin, quercetin-3- $O$-hexoside, quercetin-3-O-pentoside, kaempferol 3-O-hexoside, kaempferol 3-O-pentoside, quercetin and kaempferol.
Quercetin and kaempferol derivatives accounted for $84.8 \%$ of the total polyphenols.

Cells and reagents. The U937 human leukemic cells obtained from the American Type Culture Collection (ATCC; Manassas, VA, USA) were cultured in RPMI-1640 medium (Invitrogen Life Technologies. Carlsbad, CA, USA) supplemented with $10 \%$ (v/v) fetal bovine serum (FBS) (Gibco BRL, Grand Island, NY, USA), 1 mM L-glutamine, $100 \mathrm{U} / \mathrm{ml}$ penicillin, and $100 \mu \mathrm{g} / \mathrm{ml}$ streptomycin at $37^{\circ} \mathrm{C}$ in a humidified atmosphere of $95 \%$ air and $5 \% \mathrm{CO}_{2}$. Antibodies against $\mathrm{Bcl}-2$ (N-19), Bcl-xL, Bax, BAD, Bid, tBid, Fas, Fas ligand (FasL), Fas-associated protein with death domain (FADD), FLICE-like inhibitory protein long $\left(\mathrm{c}-\mathrm{FLIP}_{\mathrm{L}}\right)$, FLICE-like inhibitory protein short $\left(\mathrm{c}-\mathrm{FLIP}_{\mathrm{S}}\right), \mathrm{X}$-linked inhibitor of apoptosis protein (XIAP), cellular inhibitor of apoptosis protein-1 (cIAP-1), cIAP-2, procaspase-3, procaspase-8, procaspase-9, cytochrome $c$, COX IV, Akt 1/2/3 (H-136), ERK and phospho-ERK (E-4) were purchased from Santa Cruz Biotechnology Inc. (Dallas, TX, USA). Antibodies against phospho-Akt (Ser473) and phospho-Akt (Thr 308) were purchased from Cell Signaling Technology, Inc. (Beverly, MA, USA). Antibody against poly(ADP-ribose) polymerase (PARP) was purchased from BD Biosciences Pharmingen (San Diego, CA, USA). An antibody against $\beta$-actin was purchased from Sigma (Beverly, MA, USA). Peroxidase-labeled donkey anti-rabbit and sheep anti-mouse immunoglobulin, and an enhanced chemiluminescence (ECL) kit were purchased from Amersham (Waltham, MA, USA). All other chemicals not specifically cited here were purchased from Sigma-Aldrich (St. Louis, MO, USA). All these solutions were stored at $-20^{\circ} \mathrm{C}$.

Cell viability assay. The cell viability was assessed by an MTT assay. Briefly, U937 cells were treated with PES for $24 \mathrm{~h}$, and then incubated in $0.5 \mathrm{mg} / \mathrm{ml} \mathrm{3-(4,5-dimethylthi-}$ azol-2-yl)-2,5-diphenyltetrazolium bromide $(0.5 \mathrm{mg} / \mathrm{ml})$ solution for $3 \mathrm{~h}$ at $37^{\circ} \mathrm{C}$ in the dark. The absorbance of each well was measured at $540 \mathrm{~nm}$ with an enzyme-linked immunosorbent assay (ELISA) reader (Molecular Devices, LLC, Sunnyvale, CA, USA).

Nuclear staining. U937 cells treated with PES at the indicated concentrations for $24 \mathrm{~h}$ were harvested, washed with phosphate-buffered saline (PBS), and fixed with $3.7 \%$ paraformaldehyde in PBS for $10 \mathrm{~min}$ at room temperature. After being washed with PBS, the cells were stained with $2.5 \mu \mathrm{g} / \mathrm{ml}$ 4',6-diamidino-2-phenylindole (DAPI) solution for $10 \mathrm{~min}$ at room temperature. The cells were washed two more times with PBS, and observed under a fluorescence microscope.

Agarose gel electrophoresis for DNA fragmentation test. The cells treated by PES at the indicated concentrations for $24 \mathrm{~h}$ were harvested, and lysed in a buffer containing $10 \mathrm{mM}$ Tris- $\mathrm{HCl}$ ( $\mathrm{pH} \mathrm{7.4),} 150 \mathrm{mM} \mathrm{NaCl}, 5 \mathrm{mM}$ EDTA, and $0.5 \%$ Triton $\mathrm{X}-100$ for $1 \mathrm{~h}$ at room temperature. The lysates were vortexed and centrifuged at 14,000 rpm for $30 \mathrm{~min}$ at $4^{\circ} \mathrm{C}$. A $25: 24: 1(\mathrm{v} / \mathrm{v} / \mathrm{v})$ equal volume of neutral phenol:chloroform:isoamyl alcohol was used for extraction of the DNA from the supernatant. Electrophoretic analysis 
was performed on $1.5 \%$ agarose gels containing $0.1 \mu \mathrm{g} / \mathrm{ml}$ ethidium bromide (EtBr).

Flow cytometric analysis for cell cycle distribution. The cells treated with PES at the indicated concentrations for $24 \mathrm{~h}$ were collected, washed with cold PBS, and then centrifuged. The pellet was fixed in $75 \%(\mathrm{v} / \mathrm{v})$ ethanol for $1 \mathrm{~h}$ at $4^{\circ} \mathrm{C}$. The cells were washed once with PBS, and resuspended in cold PI solution $(50 \mu \mathrm{g} / \mathrm{ml})$ containing RNase A $(0.1 \mathrm{mg} / \mathrm{ml})$ in PBS ( $\mathrm{pH} \mathrm{7.4)} \mathrm{for} 30 \mathrm{~min}$ in the dark. Flow cytometric analyses were performed using Beckman Coulter Cytomics FC 500 (Beckman Coulter, Inc., San Jose, CA, USA). The sub-G1 population was calculated to estimate the apoptotic cell population.

Measurement of mitochondrial membrane potential (MMP, $\Delta \Psi m)$. MMP $(\Delta \Psi \mathrm{m})$ in living U937 cells treated with PES at the indicated concentrations for $24 \mathrm{~h}$ was measured by flow cytometry with the lipophilic cationic probe JC-1, a ratiometric, dual-emission fluorescent dye. There are two excitation wavelengths, $527 \mathrm{~nm}$ (green) for the monomer form and $590 \mathrm{~nm}$ (red) for the J-aggregate form. The cells were harvested and resuspended in $500 \mu \mathrm{l}$ of PBS and incubated with $10 \mu \mathrm{M} \mathrm{JC}-1$ for $20 \mathrm{~min}$ at $37^{\circ} \mathrm{C}$. The green fluorescence reflecting the amount of damaged mitochondria was quantified by a FACS flow cytometer.

Western blot analysis. The U937 cells treated with PES at the indicated concentrations for $24 \mathrm{~h}$ were harvested and lysed. Their proteins were quantified using the Bio-Rad protein assay kit (Bio-Rad Laboratories, Inc., Hercules, CA, USA). We separated the mitochondrial fraction from whole cell lysates using a mitochondria isolation kit for cultured cells from Thermo Fisher Scientific (Waltham, MA USA). The proteins of the extracts were resolved by electrophoresis, electrotransferred to a polyvinylidene difluoride membrane from Millipore Corp. (Bedford, MA, USA), and then the membrane was incubated with the primary antibodies followed by a conjugated secondary antibody to peroxidase. Blots were developed under an ECL detection system.

Assay of caspase activity. Caspase activity was measured in PES-treated U937 cells after $24 \mathrm{~h}$ by colorimetric assay kits, which utilized the following synthetic tetra-peptides, labeled with p-nitroaniline (pNA): Asp-Glu-Val-Asp (DEAD) for caspase-3, Ile-Glu-Thr-Asp (IETD) for caspase- 8 and Leu-Glu-His-Asp (LEHD) for caspase-9. The cells were lysed in the supplied lysis buffer. The supernatants were collected and incubated with the supplied reaction buffer containing dithiothreitol with or without substrates at $37^{\circ} \mathrm{C}$. The caspase activities were determined by absorbance at $405 \mathrm{~nm}$ using a microplate reader.

Statistical analysis. Each experiment was performed in triplicate. The results are expressed as mean \pm standard error (SEM). Significant differences were determined using the one-way analysis of variance (ANOVA) with post hoc test Newman-Keuls in the case of at least three treatment groups and Student's t-test for a two group comparison. Statistical significance was defined as $\mathrm{P}<0.05$.

\section{Results}

PES inhibits cell proliferation, and induces the apoptosis of U937 human leukemic cells. To investigate the anticancer activity of PES, U937 human leukemic cells were treated with the indicated concentrations (up to $50 \mu \mathrm{g} / \mathrm{ml}$ ) of PES for $24 \mathrm{~h}$. The growth of U937 cells was inhibited by PES treatment in a dose-dependent manner, and the $\mathrm{IC}_{50}$ value was $\sim 38 \mu \mathrm{g} / \mathrm{ml}$ (Fig. 1A). To investigate further the mechanism of cell death of the U937 cells, we performed cell cycle analysis to assess the effects of PES on cell death as well as the cell cycle. As shown in Fig. 1B, PES induced significant accumulation of cells with sub-G1 DNA content (apoptotic cell population) in a dose-dependent manner. DAPI staining for nuclear morphological changes revealed that PES induced nuclear condensation and fragmentation in a dose-dependent manner (Fig. 1C). In addition, DNA fragmentation test also revealed a typical ladder pattern of DNA fragmentation which indicates internucleosomal cleavage associated with apoptosis (Fig. 1D). These findings suggest that PES induced the apoptosis of U937 the human leukemic cells.

PES induces caspase activation, and subsequent cleavage in PARP. Next, we determined whether PES-induced apoptosis was caspase-dependent. Western blot analysis revealed that PES activated procaspase-8, and procaspase- 3 as well as procaspase-9, and cleaved PARP in a dose-dependent manner (Fig. 2A). In addition, caspase activity assay revealed that PES activated caspase-3, -8 and -9 in a dose-dependent manner (Fig. 2B). In addition, as shown in Fig. 3A-D, caspase-3 inhibitor (z-DEVD-fmk, $50 \mu \mathrm{M}$ ) suppressed the PES-induced apoptosis. These findings suggest that PES induced apoptosis at least in part through the extrinsic pathway of apoptosis in the U937 cells.

PES induces apoptosis by upregulating death receptor, Fas and suppressing anti-apoptotic protein, c-FLIP, and augmented apoptosis by modulating Bcl-2 and IAP family members. To determine how the extrinsic apoptotic pathway is involved in the PES-induced apoptosis, we measured the

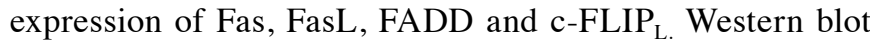
analysis revealed that PES upregulated Fas in a dose-dependent manner, and suppressed c-FLIP $\mathrm{L}_{\mathrm{L}}$ without suppressing c-FLIP $_{S}$ (Fig. 4A). These findings indicate that PES also induced the death receptor-mediated apoptosis through the extrinsic pathway. We next assessed the levels of Bcl-2 family members by western blot analysis, which revealed that PES activated Bid protein and downregulated anti-apoptotic protein, Bcl-xL (Fig. 4B). In addition, we tested the expression of IAP family members which also play an important role in caspase-dependent apoptosis. Western blot analysis revealed that PES significantly suppressed survivin, XIAP and cIAP-1 in a dose-dependent manner (Fig. 4B). These findings suggest that PES induced apoptosis by upregulating death receptor Fas and suppressing anti-apoptotic protein cFLIP, and augmented apoptosis by modulating Bcl-2 and IAP family members.

PES induces loss of MMP $(\Delta \Psi m)$ and cytochrome c release. Bcl-2 family members play an important role in inducing apoptosis by disrupting the MMP $(\Delta \Psi \mathrm{m})$ in response to 
A

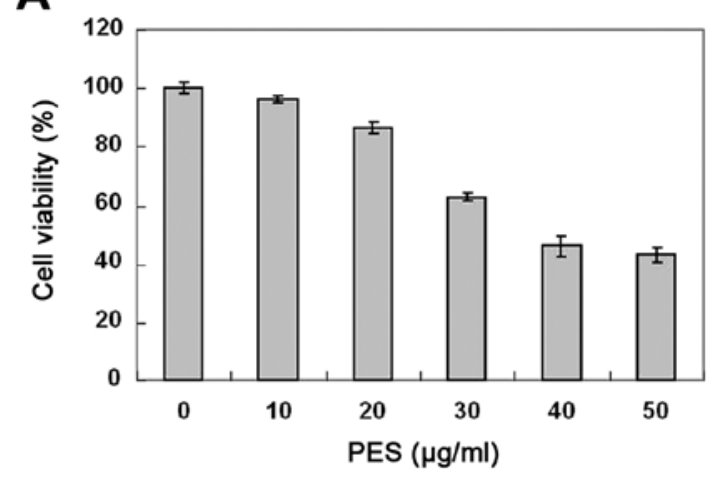

C

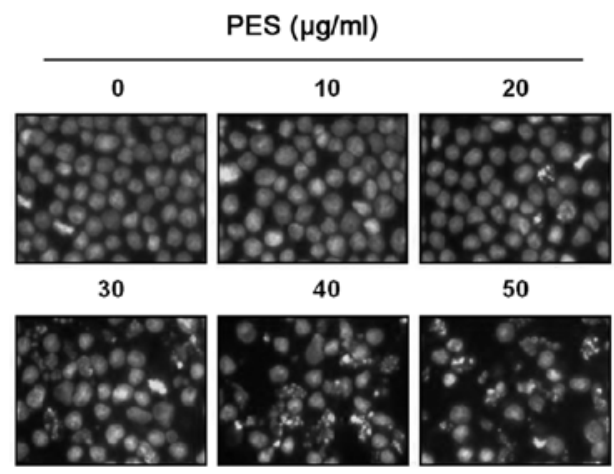

B

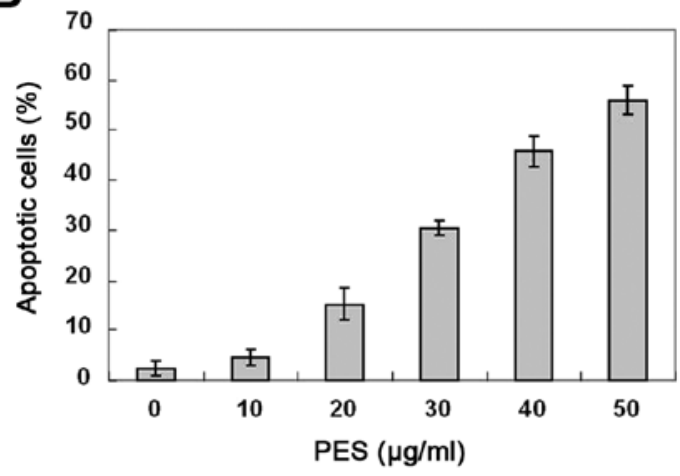

D

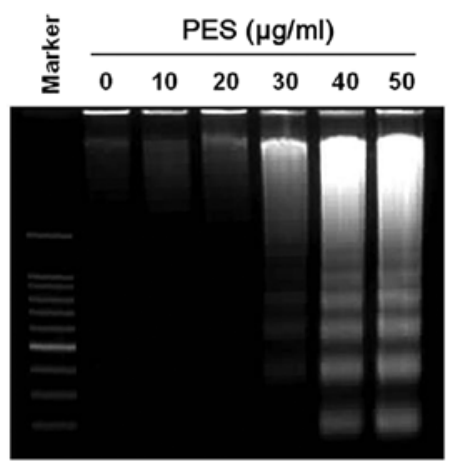

Figure 1. Growth inhibition by PES in U937 human leukemic cells. U937 cells were incubated at the indicated concentrations of PES for 24 h. (A) The growth inhibition and cytotoxicity of PES were dose-dependent manner. (B) Cell cycle analysis. The cells harboring sub-G1 DNA content represent the fraction undergoing apoptotic DNA degradation by PES treatment. (C) After fixation, the cells were stained with 4',6-diamidino-2-phenylindole (DAPI) solution to observe apoptotic bodies, which were more frequently observed at higher doses. Stained nuclei were then observed under fluorescence microscopy using a blue filter (magnification, x400). (D) DNA fragmentation test. A ladder pattern of DNA fragmentation indicates internucleosomal cleavage associated with apoptosis. The data are shown as means \pm SD of three independent experiments. ${ }^{*} \mathrm{P}<0.05$ vs. control. PES, polyphenols from Korean $E$. supina.

A

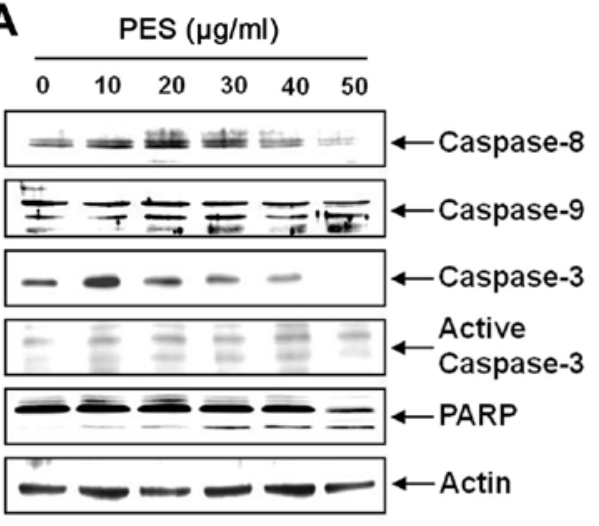

B

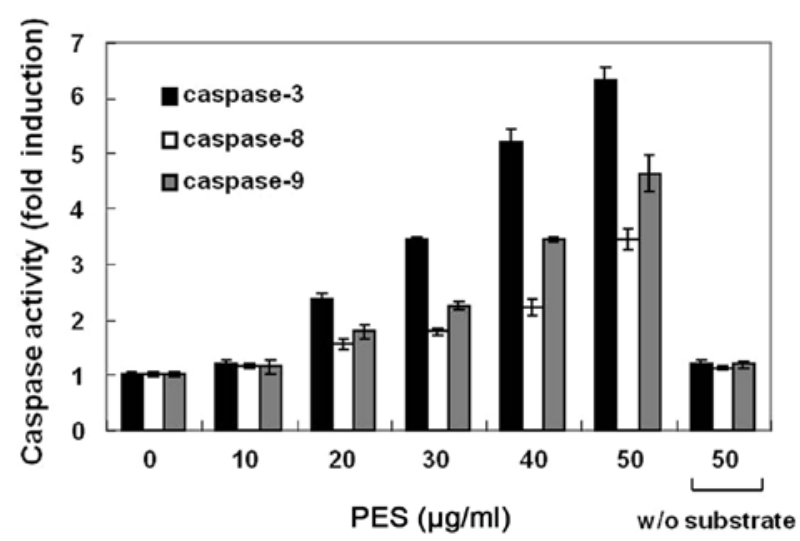

Figure 2. Activation of caspases and subsequent cleavage of poly(ADP-ribose) polymerase (PARP) during PES-induced apoptosis in U937 cells. U937 cells were incubated at the indicated concentrations of PES for $24 \mathrm{~h}$. (A) Western blot analysis of the effects of PES on the caspase activation and PARP cleavage in a dose-dependent manner. (B) In vitro assay for caspase-3, -8 and -9 activity using Asp-Glu-Val-Asp (DEVD)-pNA, Ile-Glu-Thr-Asp (IETD)-pNA and Leu-Glu-His-Asp (LEHD)-pNA as substrates, respectively. The released fluorescent products were measured. The data are shown as means \pm SD of three independent experiments. ${ }^{*} \mathrm{P}<0.05$ vs. control. PES, polyphenols from Korean E. supina.

apoptotic signaling (23). We next measured changes in MMP $(\Delta \Psi \mathrm{m})$ after PES treatment. As shown in Fig. 5A, PES induced loss of MMP $(\Delta \Psi \mathrm{m})$ in a dose-dependent manner. The loss of MMP $(\Delta \Psi \mathrm{m})$ causes the opening of the mitochondrial voltage-dependent anion channel (VDAC) (24), which results in the release of cytochrome $c$, and activation of caspases. Western blot analysis showed that PES induced cytochrome $c$ release from mitochondria (Fig. 5B). These findings suggest that the mitochondrial pathway is also important in PES-induced apoptosis. 
A

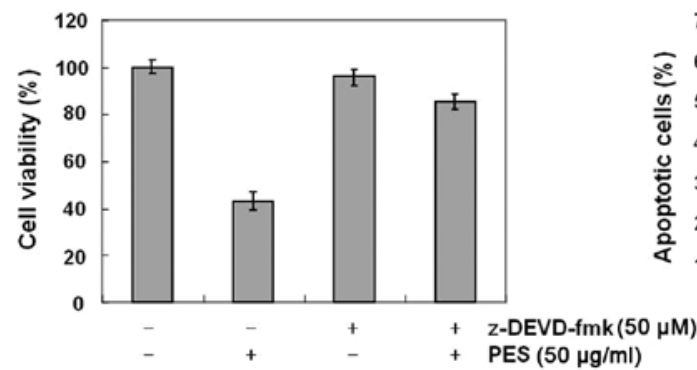

B

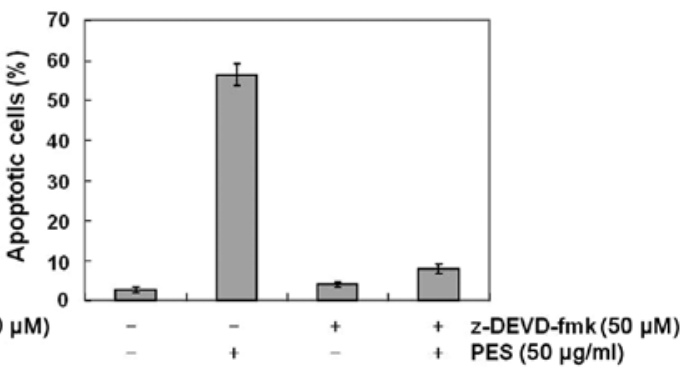

D
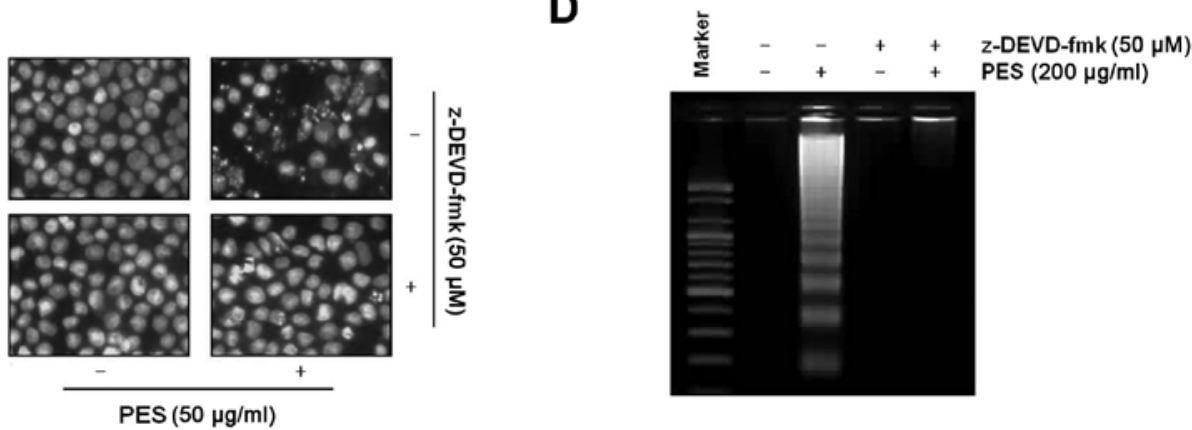

Figure 3. Role of caspase-3 in PES-induced apoptosis in U937 human leukemic cells. U937 cells were incubated at the indicated concentrations of PES with or without caspase-3 inhibitor z-DEVD-fmk $(50 \mu \mathrm{M})$. (A) The growth inhibition of PES was suppressed by z-DEVD-fmk. (B) Cell cycle analysis. The accumulation of sub-G1 DNA content in cells was decreased by z-DEVD-fmk. (C) 4',6-Diamidino-2-phenylindole (DAPI) staining. The number of apoptotic bodies was decreased by z-DEVD-fmk. Stained nuclei were observed under a fluorescence microscope using a blue filter (magnification, $x 400$ ). (D) DNA fragmentation test. The data are shown as means $\pm \mathrm{SD}$ of three independent experiments. ${ }^{*} \mathrm{P}<0.05$ vs. control; ${ }^{*} \mathrm{P}<0.05$ vs. $\mathrm{PES}(50 \mu \mathrm{g} / \mathrm{ml})$ alone treated group. PES, polyphenols from Korean E. supina.

A

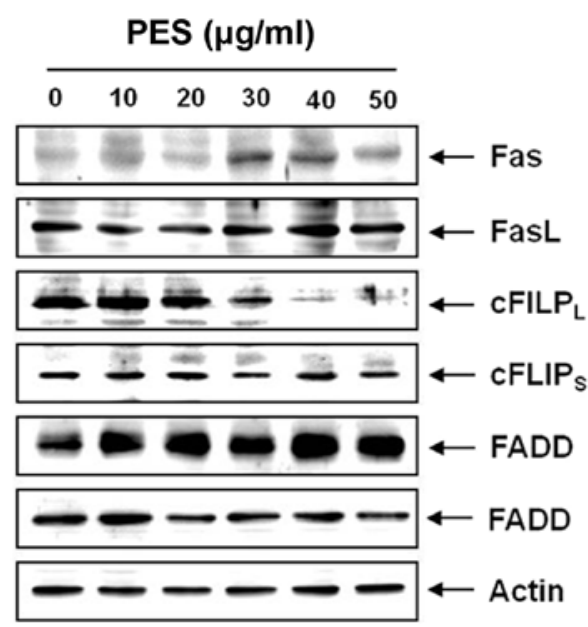

B

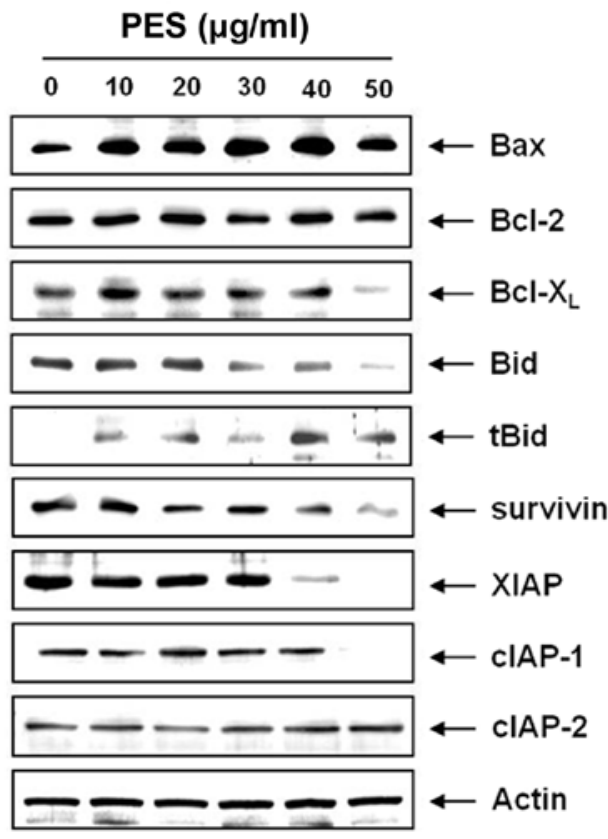

Figure 4. PES induces apoptosis by upregulating death receptor Fas and suppressing anti-apoptotic protein, c-FLIP, and augmented apoptosis by modulating Bcl-2 and inhibitor of apoptosis protein (IAP) family members. U937 cells were incubated at the indicated concentrations of PES for $24 \mathrm{~h}$. (A) Western blot analysis of the effects of PES on the U937 cells. PES upregulated Fas receptor (Fas) in a dose-dependent manner. (B) PES activated Bid, modulated the Bcl-2 family and downregulated X-linked inhibitor of apoptosis protein (XIAP), cellular inhibitor of apoptosis protein-1 (cIAP-1) and cIAP-2 in a dose-dependent manner. The results are from one representative of three independent experiments that showed similar patterns. PES, polyphenols from Korean E. supina.

PES induces apoptosis at least in part by inhibiting the phosphatidylinositol 3-kinase (PI3K)/Akt signaling pathway. The PI3K/Akt pathway plays an important role in regulating apoptosis and cell death. In addition, the expression levels of Fas and cFLIP are regulated by Akt activity $(25,26)$. Hence, we investigated the effects of PES on Akt activity in the 
A

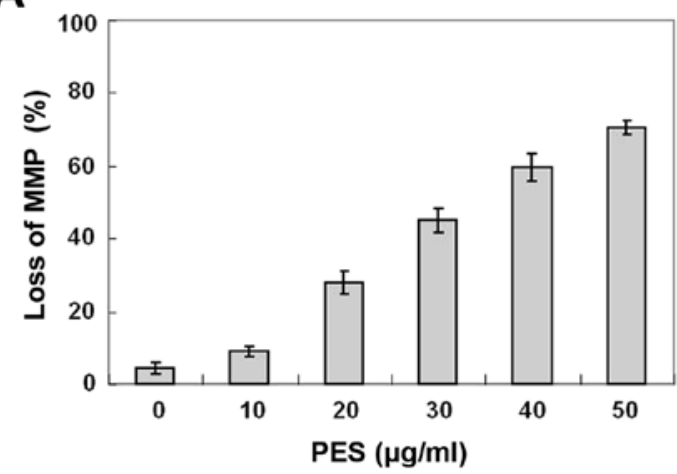

B

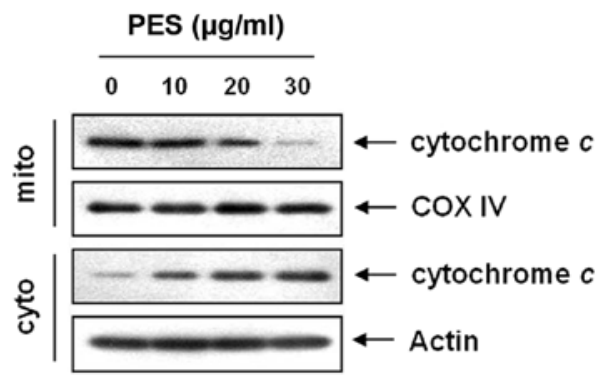

Figure 5. Effects of PES on mitochondrial membrane potential $(\Delta \Psi \mathrm{m})$ and cytochrome $c$ release in U937 cells. (A) PES induced loss of MMP $(\Delta \Psi \mathrm{m})$ in a dose-dependent manner. The cells were stained with JC-1 and incubated at $37^{\circ} \mathrm{C}$ for $30 \mathrm{~min}$. The mean JC-1 fluorescence intensity was assessed by a flow cytometer. (B) Cytochrome $c$ was released from the mitochondria. The results are from one representative of three independent experiments that showed similar patterns. ${ }^{*} \mathrm{P}<0.05$ vs. control. PES, polyphenols from Korean E. supina.

U937 cells. Since the activity of Akt is regulated by phosphorylation, we assessed the effects of PES on the levels of phosphorylated Akt in the PES-treated U937 cells. Western blot analysis revealed that PES suppressed the phosphorylation of Akt in a dose- and time-dependent manner (Fig. 6A and $\mathrm{B})$. To confirm this finding, we evaluated the effects of PES with and without treatment of the the PI3K/Akt inhibitor (LY294002). Expectedly, flow cytometric analysis for the sub-G1 fraction revealed that LY294002 augmented the effect of PES on apoptosis (Fig. 6C). These findings suggest that PES induced apoptosis at least in part by inhibiting the PI3K/Akt signaling pathway.

Activation of ERK and MAPK contributes to PES-induced apoptosis to a greater extent than suppression of PI3K/ Akt signaling. MAPK pathways are also deeply involved in cell proliferation, differentiation and apoptosis (27). Next, to further investigate the underlying mechanism responsible for PES-induced apoptosis, we assessed the changes in phosphorylation of MAPK at various concentrations of PES for $24 \mathrm{~h}$. Western blot analysis revealed that PES increased p-ERK and p-p38 MAPK in the U937 cells (Fig. 7A and B). Then we assessed the effects of MAPK inhibitors [ERK inhibitor (PD98059), JNK inhibitor (SP600125), and p38 inhibitor (SB203580)] on PES-induced apoptosis in the U937 cells. As shown in Fig. 7C-E, ERK inhibitor (PD98059) suppressed the PES-induced apoptosis, while JNK
A

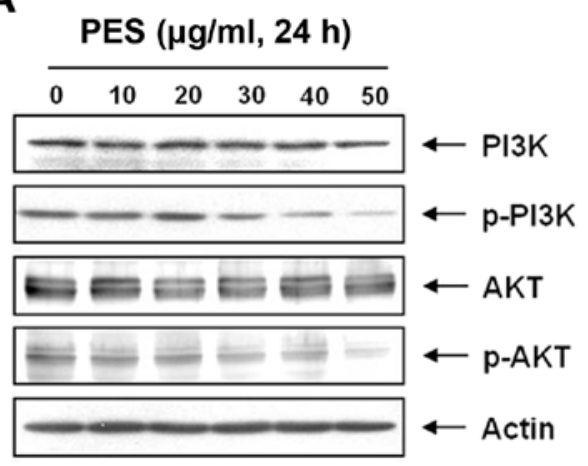

Time (24 h)

B

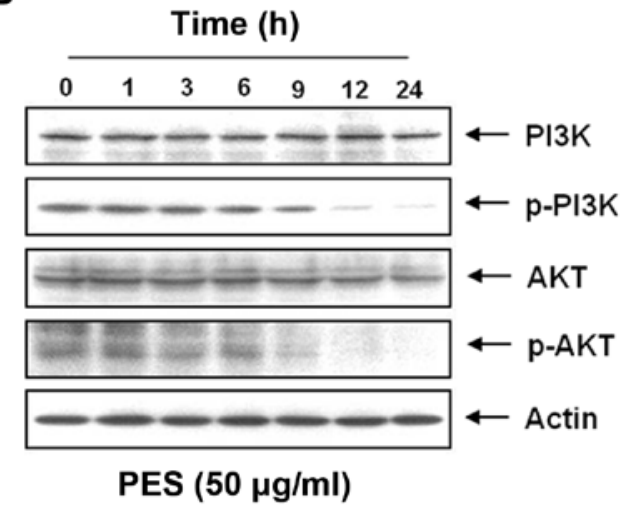

C

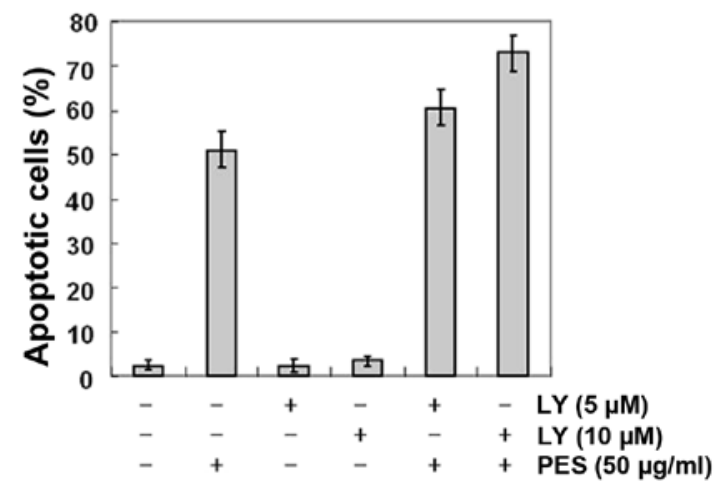

Figure 6. PES induces apoptosis at least in part by inhibiting the phosphatidylinositol 3-kinase (PI3K)/Akt signaling pathway. U937 cells were incubated at the indicated concentrations of PES for $24 \mathrm{~h}$. (A and B) Western blot analysis showed that PES inhibited PI3K/Akt expression in a dose- and time-dependent manner in the U937 cells. (C) U937 cells were incubated at the indicated concentrations of PES with or without the PI3K/Akt (LY294002) inhibitor (LY). LY294002 moderately increased PES-induced apoptosis in the U937 cells. The results are from one representative of three independent experiments that showed similar patterns. PES, polyphenols from Korean E. supina.

inhibitor (SP600125), and p38 inhibitor (SB203580) did not. This finding suggests that PES induced apoptosis at least in part by activation of ERK.

Finally, using DAPI staining (Fig. 8A) and DNA fragmentation test (Fig. 8B), and PARP cleavage (Fig. 8C), we also confirmed the effects of PI3K/Akt and MAPK on PES-induced 
A

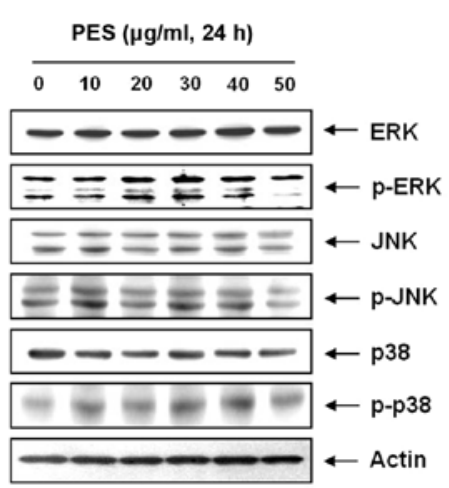

C

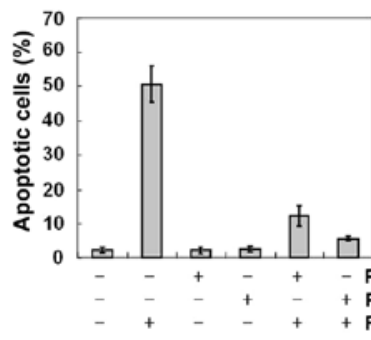

D
B

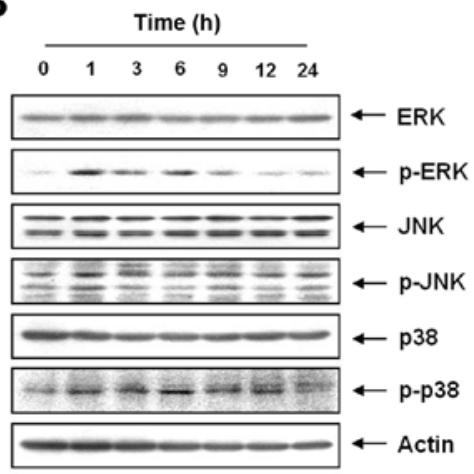

E

Figure 7. The role of MAPKs in the PES-induced apoptosis of U937 cells. U937 cells were incubated at indicated concentrations of PES for $24 \mathrm{~h}$. (A and B) Western blot analysis revealed that PES increased p-ERK and p-p38 MAPK in a dose- and time-dependent manner in U937 cells. (C-E) Cell cycle analysis shows the accumulation of subG1 DNA content. ERK inhibitor (PD98059) (PD) suppressed the PES-induced apoptosis, while JNK inhibitor (SP600125) (SP), and p38 inhibitor (SB203580) (SB) did not. The results are from one representative of three independent experiments that showed similar patterns. PES, polyphenols from Korean E. supina; MAPKs, mitogen-activated protein kinases.

A

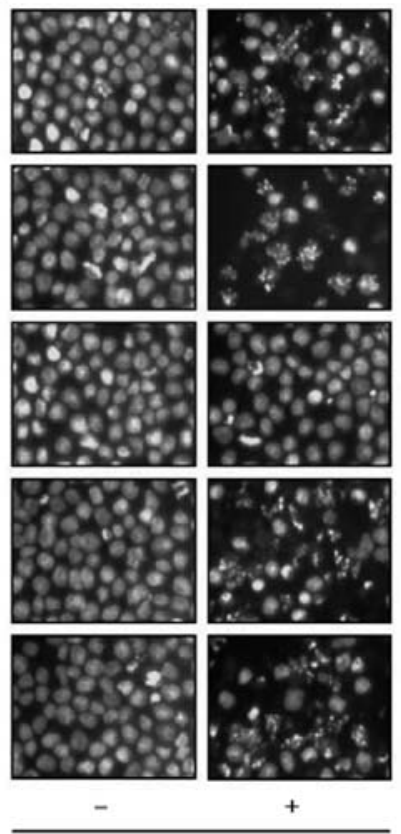

$\operatorname{PES}(50 \mu \mathrm{g} / \mathrm{ml})$
B

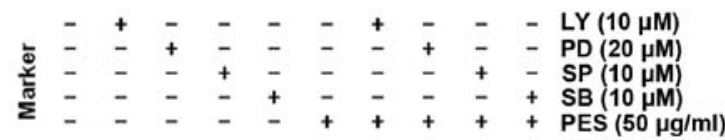

$\operatorname{LY}(10 \mu \mathrm{M})$

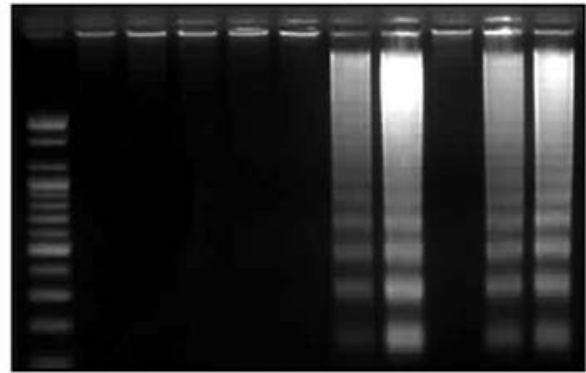

$\mathrm{SP}(10 \mu \mathrm{M}) \quad \mathrm{C}$

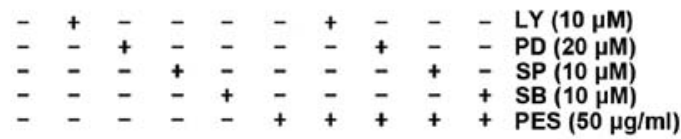

$\mathrm{SB}(10 \mu \mathrm{M})$

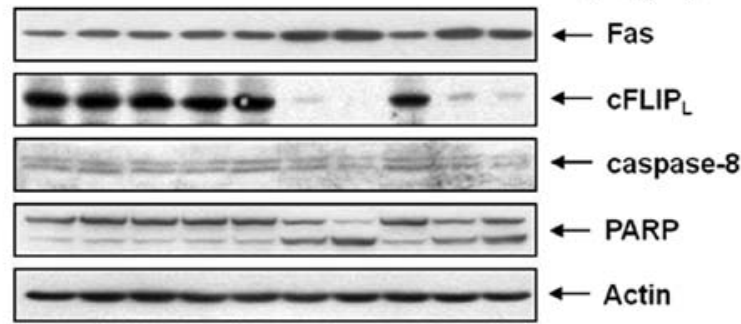

Figure 8. Activation of ERK and MAPK contributes to PES-induced apoptosis to a greater extent than suppression of PI3K/Akt signaling. U937 cells were incubated at indicated concentrations of PES with or without the PI3K/Akt (LY294002) inhibitor, the ERK inhibitor (PD98059), the JNK inhibitor (SP600125), and the p38 inhibitor (SB203580). The results showed that although PES suppressed PI3K/Akt signaling, the activation of ERK contributed more to PES-induced apoptosis rather than PI3K/Akt signaling suppression. (A) 4',6-Diamidino-2-phenylindole (DAPI) staining, (B) DNA fragmentation test and (C) western blot analysis. The results are from one representative of three independent experiments that showed similar patterns. PES, polyphenols from Korean E. supina; LY, LY294002; PD, PD98059; SP, SP600125; SB, SB203580. 
apoptosis of the U937 cells. This finding suggests that although PES suppressed PI3K/Akt signaling, the activation of ERK contributed to a greater extent to PES-induced apoptosis rather than suppression of PI3K/Akt signaling.

\section{Discussion}

The present study was conducted to elucidate the molecular mechanisms of PES-induced apoptosis in U937 human leukemic cells. The results of this study suggest that PES induced apoptosis via both the intrinsic and extrinsic pathways. Firstly, PES strongly inhibited the growth of U937 human leukemic cells in a dose-dependent manner (Fig. 1A). Furthermore, PES induced caspase activation and subsequent cleavage of PARP. The capase-dependent cell death was involved in Fas upregulation and suppression of c-FLIP . These two molecules are closely associated with PI3K/Akt activity $(25,26)$. In addition, PES suppressed the phosphorylation of PI3K and Akt which meant suppression of PI3K/Akt activity. In addition, we demonstrated that the PI3K/Akt inhibitor,LY294002, augmented PES-induced apoptosis in the U937 cells. These findings suggest that PES can induce apoptosis at least in part by inhibiting the PI3K/Akt signaling pathway. MAPK pathways are also deeply involved in cell proliferation, differentiation and apoptosis (27). Our results showed that PES increased p-ERK and p-p38 MAPK in the U937 cells. Among MAPKs, the ERK inhibitor (PD98059) suppressed the PES-induced apoptosis. These findings also suggest that activation of ERK was involved in PES-induced apoptosis in the U937 cells. Furthermore, PES-induced caspase-8 activation led to activation of pro-apoptotic protein Bid (Fig. 4B). In turn, this activated tBid translocation from the cytoplasm to the mitochondria, which increased mitochondrial permeability, and finally released cytochrome $c$ which then activated the caspase cascade for apoptosis (28). Consistent with the above results, loss of MMP was significantly increased in a dose-dependent manner (Fig. 5A). Furthermore, PES decreased the expression of Bcl-xL, XIAP, and cIAP-1 (Fig. 4B) which are direct inhibitors of activated caspases (29). This finding that PES suppressed anti-apoptotic factors such as Bcl-xL, XIAP and cIAP-1 suggest that the intrinsic pathway of apoptosis is also important in PES-induced apoptosis. These findings are similar to those in our previous study demonstrating that polyphenols extracted from Allium cepa induced apoptosis in human leukemia cells U937, through the extrinsic pathway by upregulating DR5 as well as through the intrinsic pathway by modulating Bcl-2 and IAP family members (30). Altogether, our study suggests that PES induce caspase-dependent apoptosis via both the intrinsic and extrinsic pathways. Finally, we tested whether PI3K/Akt and/or MAPK are involved in PES-induced apoptosis. These results suggest that although PES suppressed PI3K/Akt signaling, the activation of ERK contributed more to PES-induced apoptosis rather than suppression of PI3K/Akt signaling.

In conclusion, this study demonstrated the mechanisms involved in the anticancer effects of PES on U937 human leukemic cells. PES induced apoptosis via both the intrinsic and extrinsic apoptotic signaling pathways; PES upregulated Fas, activated Bid, suppressed $\mathrm{cFLIP}_{\mathrm{L}}$, and modulated the Bcl-2 family and the IAP family proteins, finally inducing apoptosis through activation of caspases. In addition, the PES-induced apoptosis was at least in part associated with ERK activation. Our findings provide the underlying molecular mechanisms for the anticancer activity of PES that can be useful in the treatment of leukemia.

\section{Acknowledgements}

This study was supported by grants from the National R\&D Program for Cancer Control, Ministry of Health \& Welfare, Republic of Korea (no. 0820050).

\section{References}

1. Lee JE, Männistö S, Spiegelman D, Hunter DJ, Bernstein L, van den Brandt PA, Buring JE, Cho E, English DR, Flood A et al: Intakes of fruit, vegetables, and carotenoids and renal cell cancer risk: a pooled analysis of 13 prospective studies. Cancer Epidemiol Biomarkers Prev 18: 1730-1739, 2009.

2. Gandini S, Merzenich H, Robertson C and Boyle P: Meta-analysis of studies on breast cancer risk and diet: the role of fruit and vegetable consumption and the intake of associated micronutrients. Eur J Cancer 36: 636-646, 2000.

3. Hatcher H, Planalp R, Cho J, Torti FM and Torti SV: Curcumin: from ancient medicine to current clinical trials. Cell Mol Life Sci 65: 1631-1652, 2008.

4. Jung KW, Won YJ, Kong HJ, Oh CM, Lee DH and Lee JS: Cancer statistics in Korea: incidence, mortality, survival, and prevalence in 2011. Cancer Res Treat 46: 109-123, 2014.

5. Jung KW, Won YJ, Kong HJ, Oh CM, Lee DH and Lee JS: Prediction of cancer incidence and mortality in Korea, 2014. Cancer Res Treat 46: 124-130, 2014.

6. Song Y, Jeong SW, Lee WS, Park S, Kim YH, Kim GS, Lee SJ, Jin JS, Kim CY, Lee JE, et al: Determination of polyphenol components of Korean prostrate spurge (Euphorbia supina) by using liquid chromatography-tandem mass spectrometry: overall contribution to antioxidant activity. J Anal Methods Chem 2014: 418690, 2014.

7. Rice EL: Inhibition of nitrogen-fixing and nitrifying bacteria by seed plants: VI. Inhibitors from Euphorbia supina. Physiol Plant 22: 1175-1183, 1969.

8. Cai WH, Matsunami K and Otsuka H: Supinaionosides A and B: megastigmane glucosides and supinanitrilosides A-F: hydroxynitrile glucosides from the whole plants of Euphorbia supina Rafinesque. Chem Pharm Bull (Tokyo) 57: 840-845, 2009.

9. Le Marchand L: Cancer preventive effects of flavonoids - a review. Biomed Pharmacother 56: 296-301, 2002.

10. Buendia B, Santa-Maria A and Courvalin JC: Caspase-dependent proteolysis of integral and peripheral proteins of nuclear membranes and nuclear pore complex proteins during apoptosis. J Cell Sci 112: 1743-1753, 1999.

11. Kerr JF, Wyllie AH and Currie AR: Apoptosis: a basic biological phenomenon with wide-ranging implications in tissue kinetics. Br J Cancer 26: 239-257, 1972.

12. Danial NN and Korsmeyer SJ: Cell death: critical control points. Cell 116: 205-219, 2004.

13. Armstrong JS: Mitochondrial membrane permeabilization: the sine qua non for cell death. BioEssays 28: 253-260, 2006.

14. Lemasters JJ, Qian T, He L, Kim JS, Elmore SP, Cascio WE and Brenner DA: Role of mitochondrial inner membrane permeabilization in necrotic cell death, apoptosis, and autophagy. Antioxid Redox Signal 4: 769-781, 2002.

15. Boya P, Cohen I, Zamzami N, Vieira HL and Kroemer G: Endoplasmic reticulum stress-induced cell death requires mitochondrial membrane permeabilization. Cell Death Differ 9: 465-467, 2002.

16. Li P, Nijhawan D, Budihardjo I, Srinivasula SM, Ahmad M, Alnemri ES and Wang X: Cytochrome $c$ and dATP-dependent formation of Apaf-1/caspase-9 complex initiates an apoptotic protease cascade. Cell 91: 479-489, 1997.

17. Wajant H: The Fas signaling pathway: more than a paradigm. Science 296: 1635-1636, 2002.

18. Chen G and Goeddel DV: TNF-R1 signaling: a beautiful pathway. Science 296: 1634-1635, 2002.

19. Walczak H and Krammer PH: The CD95 (APO-1/Fas) and the TRAIL (APO-2L) apoptosis systems. Exp Cell Res 256: 58-66, 2000. 
20. Murphy KM, Ranganathan V, Farnsworth ML, Kavallaris M and Lock RB: Bcl-2 inhibits Bax translocation from cytosol to mitochondria during drug-induced apoptosis of human tumor cells. Cell Death Differ 7: 102-111, 2000.

21. Mitsiades CS, Mitsiades N and Koutsilieris M: The Akt pathway: molecular targets for anti-cancer drug development. Curr Cancer Drug Targets 4: 235-256, 2004.

22. Pearson G, Robinson F, Beers Gibson T, Xu BE, Karandikar M, Berman K and Cobb MH: Mitogen-activated protein (MAP) kinase pathways: regulation and physiological functions. Endocr Rev 22: 153-183, 2001.

23. Hengartner MO: The biochemistry of apoptosis. Nature 407: 770-776, 2000

24. Timmer JC and Salvesen GS: Caspase substrates. Cell Death Differ 14: 66-72, 2007.

25. Maddigan A, Truitt L, Arsenault R, Freywald T, Allonby O, Dean J, Narendran A, Xiang J, Weng A, Napper S, et al: EphB receptors trigger Akt activation and suppress Fas receptor-induced apoptosis in malignant T lymphocytes. J Immunol 187: 5983-5994, 2011.
26. Wang X, Chen W, Zeng W, Bai L, Tesfaigzi Y, Belinsky SA and Lin Y: Akt-mediated eminent expression of c-FLIP and Mcl-1 confers acquired resistance to TRAIL-induced cytotoxicity to lung cancer cells. Mol Cancer Ther 7: 1156-1163, 2008.

27. Robinson MJ and Cobb MH: Mitogen-activated protein kinase pathways. Curr Opin Cell Biol 9: 180-186, 1997.

28. Kelekar A and Thompson CB: Bcl-2-family proteins: the role of the BH3 domain in apoptosis. Trends Cell Biol 8: 324-330, 1998.

29. Hunter AM, LaCasse EC and Korneluk RG: The inhibitors of apoptosis (IAPs) as cancer targets. Apoptosis 12: 1543-1568, 2007.

30. Han MH, Lee WS, Jung JH, Jeong JH, Park C, Kim HJ, Kim G, Jung JM, Kwon TK, Kim GY, et al: Polyphenols isolated from Allium cepa L. induces apoptosis by suppressing IAP-1 through inhibiting PI3K/Akt signaling pathways in human leukemic cells. Food Chem Toxicol 62: 382-389, 2013. 